\title{
4/8 HLA Match
}

National Cancer Institute

\section{Source}

National Cancer Institute. 4/8 HLA Match. NCI Thesaurus. Code C158038.

Degree of HLA match is $4 / 8$ alleles with fewer than 2 mismatches per locus at HLA-A, -B, -C and-DRB1. 